\title{
DISCUSSION ABOUT THE USE OF BAYESIAN NETWORKS MODELS FOR MAKING PREDICTIVE MAINTENANCE DECISIONS
}

\author{
Rafaela Bortolini ${ }^{1}$ and Núria Forcada ${ }^{2}$
}

\begin{abstract}
The performance of a building decreases with time and this process is accelerated if proper maintenance is not carried out. This paper presents a discussion about the importance of predictive maintenance actions to enhance the condition of existing buildings. An approach based on Bayesian networks (BN) is proposed to predict the condition of a building. The proposed approach consists of a conceptual and generic model, including the factors with more influence in the condition of a building, which were identified by a literature review. The relationships between these factors and a discussion about the application of this model in maintenance decision-making are provided.
\end{abstract}

Keywords: Building performance, decision-making, predictive maintenance.

\section{INTRODUCTION}

Buildings tend to reduce their performance in terms of condition and increasingly fail to meet users' needs, requirements and expectations (Gaspar and Brito 2005) unless proper maintenance is carried out (Wilde et al. 2011). Maintaining the condition of a building and preventing the degradation of its elements and systems depends on decisions involving maintenance tasks, which are made based on various sources of facility data such as inspections and sensors (Chen et al. 2013). Lack of decision-making hinders the optimization of building condition and results in cost overruns by inefficient building maintenance operations associated with unnecessary interventions or urgent repairs (Silva et al. 2016). The rate of degradation of the built environment in Europe is of enormous economic and technical importance, since about half of the expenditure in the construction industry is spent on repair, maintenance and remediation (Balaras et al. 2005).

In order to minimize excessive expenses with reactive maintenance, predictive maintenance approach should be supported in nowadays Facility Management (FM) activities (Akcamete et al. 2010). Predictive maintenance is based on the asset condition with the aim to eliminate unexpected breakdowns, minimize unscheduled downtimes and consequently reduce maintenance costs $(\mathrm{Wu}$ et al. 2007). Predictive maintenance originates from the manufacturing industry and there are few reported studies that evaluate the application of this approach on building elements. Capturing reliable data about degradation factors is crucial to assess the condition of building elements. A number of parameters like the quality of the materials, the local weather conditions and the lack of maintenance can greatly influence the degradation process (Balaras et al. 2005).

$1 \quad$ PhD candidate, Department of Project and Construction Engineering, Group of Construction Research and Innovation, Universitat Politècnica de Catalunya, ES, bortolinirafaela@gmail.com

2 Associate Professor, Department of Project and Construction Engineering, Group of Construction Research and Innovation, Universitat Politècnica de Catalunya, ES, nuria.forcada@upc.edu 
The aim of this study is to provide a support in the decision-making of predictive maintenance actions, in order to enhance the condition of existing buildings. This paper reports an ongoing research project which proposes the use of Bayesian networks (BN) based approach to predict the condition of existing buildings and help predictive maintenance. $\mathrm{BN}$ is a probabilistic graphical model that represent a set of variables and their probabilistic dependencies. BN use computational model for human reasoning, namely, the mechanism by which people integrate data from multiple sources to create coherent interpretations of that data (Pearl 1988). As a starting point of this investigation, this study concentrates on the assessment of the factors that affect building condition, and how these factors are interrelated in a BN model.

\section{BACKGROUND}

\subsection{Predictive maintenance}

Maintenance management is a key branch in the extensive field of FM, as it is the focus of most FM activities. There are many maintenance activities in a building, including but not limited to actions on scheduling, procedures, work control, optimization of routine, preventive and predictive actions with the aim to increase efficiency, reliability and safety of buildings (Sullivan et al. 2010). Predictive maintenance is concentrated on detecting the system degradation and to conduct maintenance on the actual condition of the element under analysis (Sullivan et al. 2010). Once a fault is detected, the condition of the element should be monitored regularly to avoid emergencies. It has originally been used in industrial plants to predict the performance of machines by applying techniques such as: lubricant analysis, vibration analysis, thermography, penetrating liquids, radiography, and control of corrosion (Edwards et al. 1998).

In addition to machines, this approach can be applied to the elements of a building (Chen et al. 2013), which depend on the condition assessment of the building estimating the risk of the degradation process (Flores-Colen et al. 2010). Addressing risk in building condition evaluation is significant, since several studies have shown that poor building performance presents risks towards the safety and health of its users (Khalil et al. 2016). The problem is that risk is associated with many factors that are not independent and their relations are uncertain. To deal with this problem consistently and effectively, there is a need of a rigorous method for quantifying uncertainty.

\subsection{Bayesian networks}

$\mathrm{BN}$ is a powerful tool in artificial intelligence to model risk with uncertain data and have been extensively used to develop decision support systems in a variety of domains (Nguyen et al. 2016). Although it has been an attractive technique to examine a range of issues in the construction industry (Wang et al. 2014), it is still relatively new in this field (Nguyen et al. 2016). This tool is a combination of two different parts: graph theory and probability theory. BN are consisted of a directed acyclic graph (DAG) and an associated set of conditional probability tables (CPTs) (Pearl 1988). The nodes of the DAG represent random variables with a finite set of states and the edges correspond to probabilistic causal dependence among the variables (Pearl 1988). The CPT represents the conditional probability distribution between the variables. The analysis of how much a specific node is influenced by other nodes is possible by sensitivity analysis (Khodakarami and Abdi 2014). Among the advantages of BN, Uusitalo (2007) highlights the suitability for small 
and incomplete data sets, the possibility to combine different sources of knowledge and the ability to model causal relationships between variables.

\section{BN FOR BUILDING CONDITION PREDICTION}

\subsection{Identification of factors}

There have been some initiatives with different approaches to predict the condition of a building. These studies are related to degradation factors that affect building condition, and in general they are related to the technical performance of a building (Lützkendorf et al. 2005). Technical performance is related to structural, physical and well-functioning building equipment.

Some studies have analysed degradation factors related to the environmental agents. The condition of buildings depends on the climate they are exposed to (Wilde and Coley 2012; Wu et al. 2010), such as sunlight, rain, frost action, humidity, condensation, wind and pollution (Olanrewaju and Abdul-Aziz 2015). There are also factors influencing the degradation process related to building characteristics, like the quality of components and materials (Rodrigues et al. 2011; Sulakatko et al. 2014), the age of the building (FloresColen et al. 2010), the efficiency of equipment (Heo et al. 2012).

Other studies have focused on building defects, which play an important role in increasing the effectiveness of building maintenance strategies (Pereira et al. 2013). Defect is the term used to define a failing or shortcoming in the function, performance, statutory or user requirements of a building, and might manifest itself within the structure, fabric, services or other facilities of the affected building (Watt 1999). Usually, previous studies have identified defects and their probable causes with focus on a specific building element or system, such as façade (Chew et al. 2004; Pereira et al. 2013; Sulakatko et al. 2014; Serralheiro et al. 2017), wet areas (Chew et al. 2004), electrical and plumbing systems (Das and Chew 2011).

Sullivan et al. (2010) highlighted the importance of regularly cleaning and inspecting the systems in a building. For example, the deterioration of a chiller is highly associated to the contamination of tubes, which can be recovered by using chemical cleaning (Wilde et al. 2011). Inspections can aid to define the status of an equipment as well as establish appropriate actions to prevent defects (Mathew 2004). Some defects are unrevealed and can be detected only at scheduled inspections (Taghipour et al. 2010). This is related to preventive maintenance actions ruled by regulations. Mandatory building inspections ensure that a building is safe and its environment is healthy (Chan et al. 2014). Another important factor is the tracking of complaints of end users by Computerized Maintenance Management Systems (CMMS), as well as data gathered from Building Management Systems (BMS) that monitor and detect defects of mechanical and electrical equipment such as lighting, heating, ventilating and air-conditioning systems (Picon et al. 2013).

In short, the factors with more influence in the condition of buildings are related to the following categories: (1) environmental agents, (2) building properties, (3) defects/complaints and (4) building maintenance actions. A important prerequisite was to identify factors related only to the operational phase of the building lifecycle, therefore, factors related to building design or the quality of the construction work are outside the scope of this work. 


\subsection{BN model design}

A BN model involves factors that can be classified in accordance to their purposes: a trigger node is an initiating event, an event node characterizes the impact, a control node is a way to stop the trigger event, and a mitigant node helps avoid the final consequence (Fenton and Neil 2011). The factors identified in the previous section were used to design a generic $\mathrm{BN}$ model for predicting the condition of a building (Figure 1).

The model depends on the condition of the different elements and systems. The condition of an element can be determined by the defects detected in an inspection, the defects detected in BMS and the complaints gathered from CMMS. Moreover, the condition loss of an element can be mitigated by corrective and preventive maintenance actions. Environmental agents and building properties are the triggers for different defects and complaints.

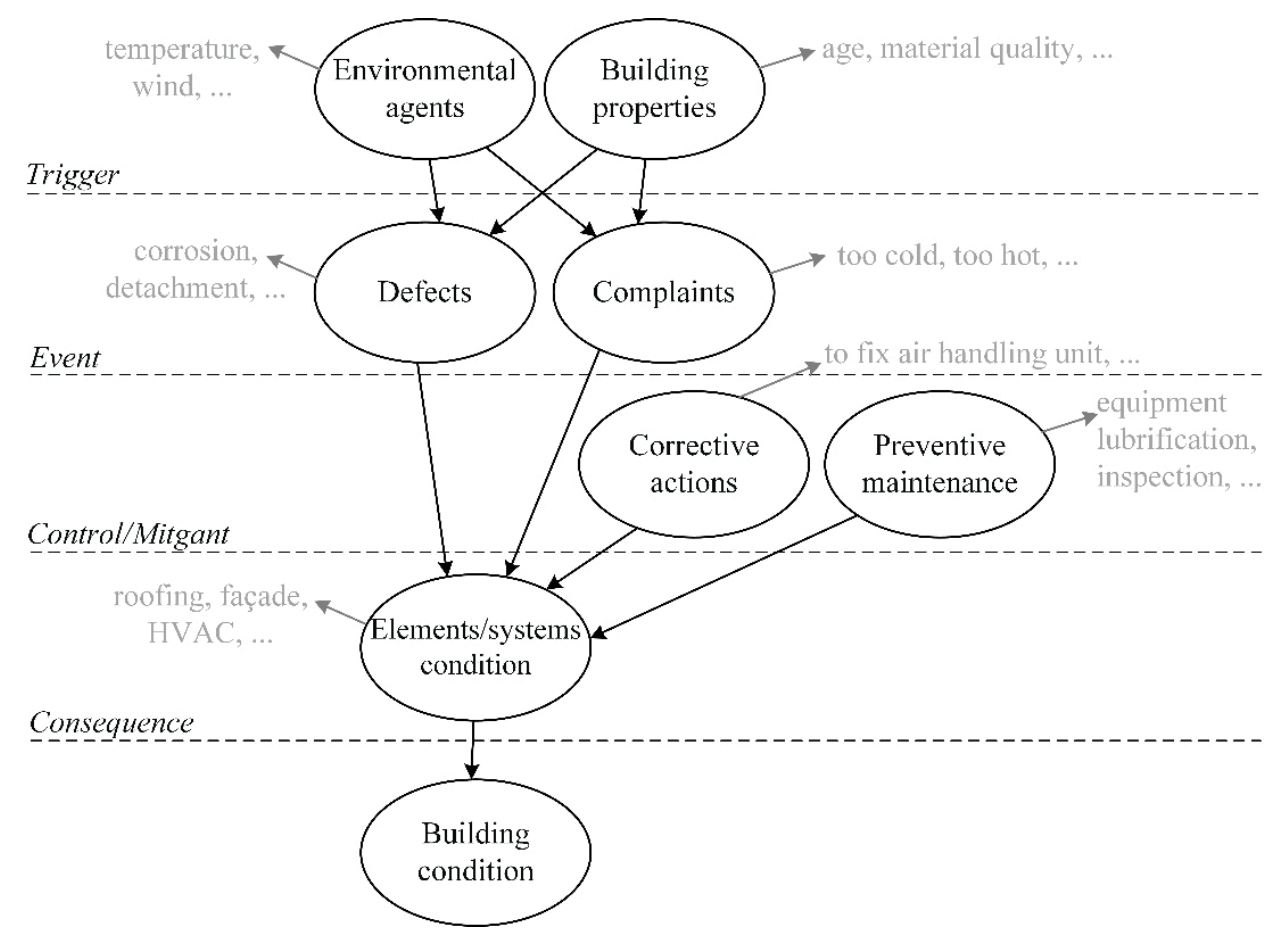

Figure 1: Generic BN model for predicting building condition

The generic BN model can be customized for a specific case, since the condition of a building depends on the condition of all building elements and systems that make up the building and the relations between them. Building elements are structure, façade, roofing, flooring and interior partitions, while building systems are HVAC, plumbing, electrical, fire systems and elevators. The BN model for a specific building should include nodes of the building elements/systems that represent such building in analysis. The classification of building elements/systems adopted in this study was based on OmniClass, which is a classification system used by COBie (Construction Operations Building Information Exchange). COBie is an international standard to manage information needed by facility managers.

Figure 2 shows an example of some relations among nodes. The façade condition depends on the severity of the cracks, detachment, etc. These defects depend on the exterior condition, age of the building, façade material quality, etc. On the other hand, other defects detected in another system such as plumbing, might affect the probability of a defect increase, i.e., a water leakage can provoke a façade detachment. Furthermore, a 
corrosion on the pipes can provoke a water leakage, which can generate a malfunctioning and affect the condition of the plumbing system. Corrective actions and preventive maintenance for each element/system can control and mitigate the final condition of the building.

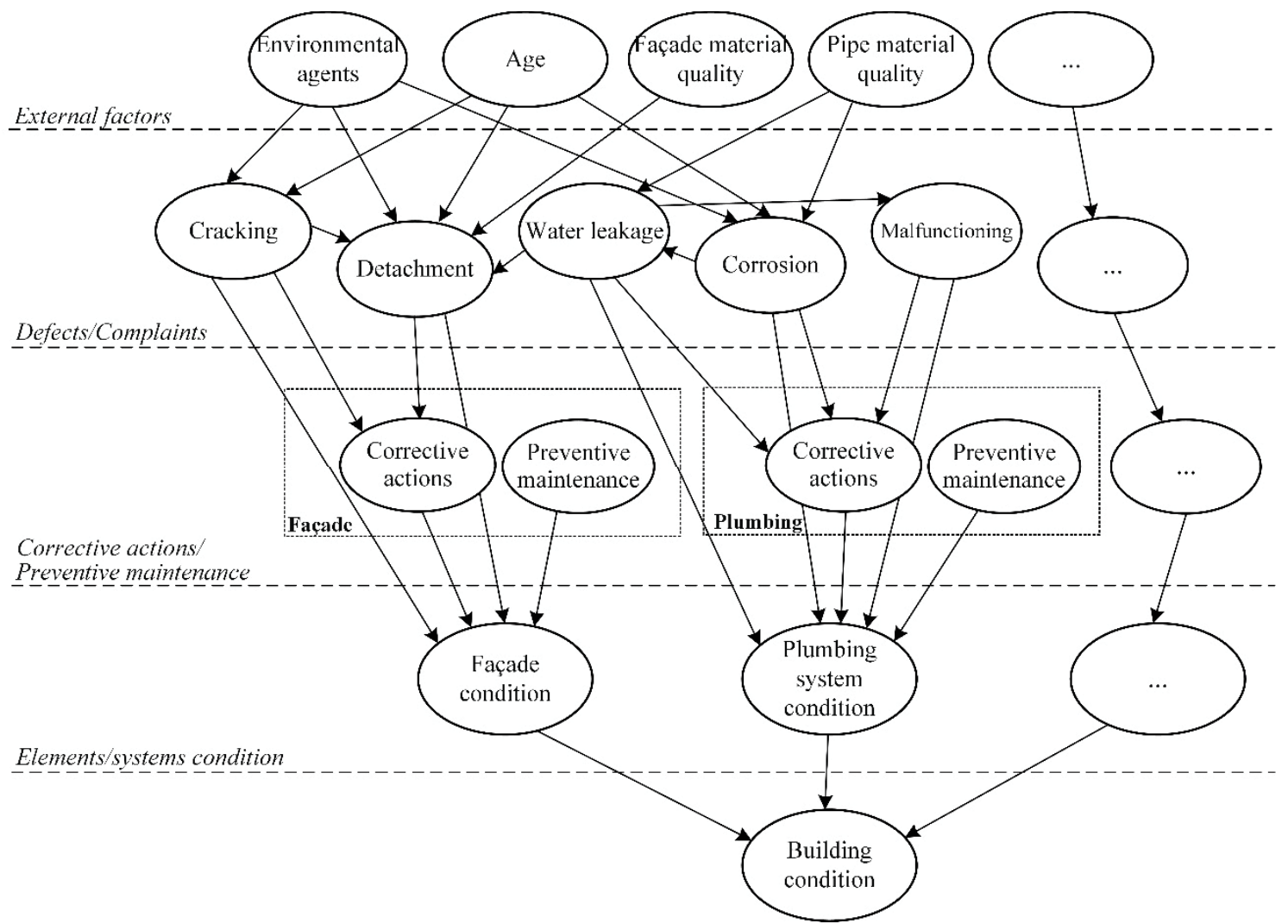

Figure 2: Example of relationship between building elements and systems

\subsection{Uncertainty analysis}

There are two different approaches to estimate the probability distributions between the nodes: (1) estimation from statistical data which is also known as parameter learning on the basis of historical data, and (2) knowledge and experience from expert judgment (Wang et al. 2014; Nguyen et al. 2016). When historic data is available, Poisson distribution can be applied into the model (Equation 1). It is a discrete probability distribution of a given number of events occurring in a fixed interval of time (Ahrens and Dieter 1974).

$$
f(x, \lambda)=\frac{e^{-\lambda} \lambda^{x}}{x !}
$$

where $\lambda$ denotes the expected number of occurrences during the given interval, i.e., the number of defects of an element or system per year on average; and $\mathrm{x}$ is the number of occurrences of an event, i.e., the number of defects of an element or system per year.

When exact values of probability are not available, an expert judgment is generally employed to assess the occurrence probability of nodes (Zhang et al. 2014). Generally, a scale is used to translate the verbal description into numerical values (Wang et al. 2014). The experts should be asked to rate the degree of influence of each factor in the condition of a building and identify critical paths through the BN model. Basically, the identified problems and their causes are included on the $\mathrm{BN}$ model to predict what is likely to happen 
making analysis of the building condition. An expert judgment and several case studies will be used to quantify the probability distributions among the variables.

\subsection{Practical implications}

In general, this model can be used to estimate the uncertainty/risk effect of degradation factors on building condition. A management review and judgment process of the results produced by the $\mathrm{BN}$ model should be done by a formal analysis. The facility manager of the building should decide about measures based on scenario analysis taking into account the resources available and the cost/benefit ratio of the actions. In the final step, the measures are reviewed and a decision of the best course of maintenance action is made.

Maintenance actions can be categorized into four types: monitor, minor repair, major repair and replacement. If an incipient defect/complaint is detected, in which there is no impact on the building condition, an action to repair it can be postponed and the defect/complaint should be monitored. Minor repair is associated with cleaning actions or doing a local repair of the deterioration. A major repair is related with actions to local replacement and preventive treatment. The replacement is associated to actions to substitute completely the material/elements when the area affected cannot be recovered. Major repair and replacement actions are highly associated with the degradation condition. When the physical integrity of the users is at risk, maintenance actions to prevent this fact must be undertaken. Moreover, based on the assessment condition of the building, regular inspections have to be planned.

It is possible to add new observations about the degradation factors on the $\mathrm{BN}$ model in order to perform different 'what if?' type analysis. Exploratory analysis can be done to evaluate if another inspection need to be carried out and make better maintenance plans by prioritizing the problems with high severity and controlling dangerous situations. Estimate the time in which inspection is needed is the main goal of predictive maintenance approach, which attempts to detect a degradation to correct it prior to significant deterioration in the element occurs. By detecting initial stages of deteriorations, the condition of a building can be protected and the costs of the maintenance interventions can be minimized.

\section{CONCLUSIONS AND FUTURE WORK}

A lack of structured methods inhibits facility managers from proactively managing buildings and reaching their maintenance. The condition of buildings is associated with various degrees of uncertainty, due to the many factors that may affect a building. These factors are generally not independent, and this work proposed the use of an approach based on $\mathrm{BN}$ to understand the relationships between these factors.

The use of BN models can yield analysis of the probability distribution of deterioration factors on buildings by case studies and expert judgment, which provide insightful information for decision makers regarding maintenance actions. This enables to express uncertainty about the final condition of a building by modelling the dependency among degradation factors and how to represent the control and mitigant of the building condition. One important advantage of $\mathrm{BN}$ is the capability of parameter learning which is updating the belief about one factor when new information is available. By entering a new observation about the degradation factors, the model automatically updates the condition of the building. 
Future steps include an investigation on the degree of impact of the variables that affect the performance of a building to validate the approach. BN has the advantage of easily incorporate more variables and modify the network to fit the problem. Additionally, the definition of the most common defects that affect each building element and system is being evaluated. Data integration from systems related to maintenance process such as BMS, CMMS and Building Information Modelling (BIM) is also being analyzed.

\section{ACKNOWLEDGMENTS}

This study was funded by the Conselho Nacional de Desenvolvimento Científico e Tecnológico (CNPq), grant 233559/2014-0.

\section{REFERENCES}

Ahrens, J. H., and Dieter, U. (1974). Computer methods for sampling from gamma, beta, poisson and bionomial distributions. Computing, 12(3), 223-246.

Akcamete, A., Akinci, B. and Garrett, J.H. (2010). Potential utilization of building information models for planning maintenance activities. Proc. of the Int. Conf. on Comp. in Civil and Build. Eng., 151-157.

Balaras, C. A., Droutsa, K., Dascalaki, E., and Kontoyiannidis, S. (2005). Deterioration of European apartment buildings. Energy and Buildings, 37(5), 515-527.

Chan, D., Hung, H., Chan, A., and Lo, T. (2014). Overview of the development and implementation of the mandatory building inspection scheme (MBIS) in Hong Kong. Built environment project and asset management, 4(1), 71-89.

Chen, H.M., Hou, C.C. and Wang, Y.H. (2013). A 3D visualized expert system for maintenance and management of existing building facilities using reliability-based method. Expert Systems with Applications, 40(1), pp.287-299.

Chew, M.Y.L. and De Silva, N. (2004). Factorial Method for Performance Assessment of Building Facades. J. Constr. Eng. Manage., 130(4), pp.525-533.

Das, S., and Chew, M. Y. (2011). Generic method of grading building defects using FMECA to improve maintainability decisions. J. of Perform. Constr. Facil., 25(6), 522-533.

Edwards, D.J., Holt, G.D. and F.C. Harris. (1998). Predictive maintenance techniques and their relevance to construction plant. J. Quality in Maint. Eng., 4(1), p.25.

Fenton, N., and Neil, M. (2011). The use of Bayes and causal modelling in decision making, uncertainty and risk. CEPIS Upgrade, 12(5), 10-21.

Flores-Colen, I., de Brito, J. and Freitas, V. (2010). Discussion of Criteria for Prioritization of Predictive Maintenance of Building Façades: Survey of 30 Experts. J. of Perform. Constr. Facil., 24(4), pp.337-344.

Gaspar, P. L., and Brito, J. D. (2005). Assessment of the overall degradation level of an element, based on field data. In 10th Int. conf. on dur. build. mat. comp., Lyon, France.

Heo, Y., Choudhary, R., and Augenbroe, G. A. (2012). Calibration of building energy models for retrofit analysis under uncertainty. Energy and Buildings, 47, 550-560.

Khalil, N., Kamaruzzaman, S. N., and Baharum, M. R. (2016). Ranking the indicators of building performance and the users' risk via Analytical Hierarchy Process (AHP): Case of Malaysia. Ecological Indicators, 71, 567-576.

Khodakarami, V. and Abdi, A. (2014). Project cost risk analysis: A Bayesian networks approach for modelling dependencies between cost items. Int. J. Proj. Manag., 32(7), pp.1233-1245. 
Lützkendorf, T., Speer, T., Szigeti, F., Davis, G., Le Roux, P., Kato, A., and Tsunekawa, K. (2005). A comparison of international classifications for performance requirements and building performance categories used in evaluation methods. Performance based building, 61-80.

Mathew, S. (2004). Optimal inspection frequency: A tool for maintenance planning/forecasting. Inter. J. of Quality \& Reliability Management, 21(7), 763-771.

Nguyen, L.D., Tran, D.Q. and Chandrawinata, M.P. (2016). Predicting Safety Risk of Working at Heights Using Bayesian Networks. J. Constr. Eng. Manag. p.04016041.

Olanrewaju, A. L., and Abdul-Aziz, A. R. (2015). Building Maintenance Processes and Practices. Springer.

Pearl, J. (1988). Probabilistic reasoning in intelligent systems: networks of plausible inference. Morgan Kaufmann Publishers Inc, San Francisco, USA.

Pereira, C., Brito, J. and Correia, J.R. (2013). Building Characterization and Degradation Condition of Secondary Industrial Schools. J. Constr. Eng. Manag., 29(5), 1-10.

Picon, L., Yannou, B., Zaraket, T., Minel, S., Bertoluci, G., Cluzel, F., and Farel, R. (2013). Use-phase memory: A tool for the sustainable construction and renovation of residential buildings. Automation in Construction, 36, 53-70.

Rodrigues, M. F. S., Teixeira, J. M., and Cardoso, J. C. (2011). Buildings envelope anomalies: A visual survey methodology. Constr. Build. Materials, 25(5), 2741-2750.

Serralheiro, M. I., de Brito, J., and Silva, A. (2017). Methodology for service life prediction of architectural concrete facades. Constr. Build. Materials, 133, 261-274.

Silva, A., de Brito, J. and Gaspar, P.L. (2016). Methodologies for Service Life Prediction of Buildings, Cham: Springer International Publishing.

Sulakatko, V., Lill, I., Soekov, E., Arhipova, R., Witt, E., and Liisma, E. (2014). Towards nearly zero-energy buildings through analyzing reasons for degradation of facades. Procedia Economics and Finance, 18, 592-600.

Sullivan, G. P., Pugh, R., Melendez, A. P., and Hunt, W. D. (2010). Operations \& Maintenance Best Practices. A guide to achieving operational efficiency, Release, 2.

Taghipour, S., Banjevic, D., and Jardine, A. K. (2010). Periodic inspection optimization model for a complex repairable system. Reliab. Eng. \& Syst. Saf., 95(9), 944-952.

Uusitalo, L. (2007). Advantages and challenges of Bayesian networks in environmental modelling. Ecological Modelling, 203(3-4), pp.312-318.

Wang, F., Ding, L. Y., Luo, H. B., and Love, P. E. (2014). Probabilistic risk assessment of tunneling-induced damage to existing properties. Exp. Syst. App., 41(4), 951-961.

Watt, D. (1999). Building pathology: Principles and practice. Blackwell Science, Oxford.

de Wilde, P., Tian, W. and Augenbroe, G. (2011). Longitudinal prediction of the operational energy use of buildings. Building and Environment, 46(8), pp.1670-1680.

Wu, S. J., Gebraeel, N., Lawley, M. A., and Yih, Y. (2007). A neural network integrated decision support system for condition-based optimal predictive maintenance policy. IEEE Trans. Systems, Man, and Cybernetics-Part A: Systems and Humans, 226-236.

Zhang, L., Wu, X., Skibniewski, M. J., Zhong, J., and Lu, Y. (2014). Bayesian-networkbased safety risk analysis in construction projects. Reliab. Eng. \& Syst. Saf., 29-39. 